\title{
The structure of titanate nanobelts used as seeds for the nucleation of hydroxyapatite at the surface of titanium implants
}

\author{
E. Conforto ${ }^{1 *}$, D. Caillard ${ }^{2}$, L. Müller ${ }^{3}$, F.A. Müller ${ }^{3}$ \\ ${ }^{1}$ Centre Commun d'Analyses, Université de La Rochelle, F-17071 La Rochelle Cedex 9, France \\ ${ }^{2}$ CEMES-CNRS, BP 4347, F-31055 Toulouse Cedex, France \\ ${ }^{3}$ University of Erlangen-Nürnberg, Department of Materials Science III - Biomaterials, \\ Henkestr. 91, 91052 Erlangen, Germany
}

\begin{abstract}
The sequence of steps of a chemical treatment having as goal to induce the nucleation and growth of hydroxyl carbonated apatite (HCA) at the surface of titanium implants was studied by scanning and transmission electron microscopy, in cross-section. In the first step, an acid etching forms a rough titanium hydride layer, which remains unchanged after subsequent treatments. In a second step, soaking in a $\mathrm{NaOH}$ solution induces the growth of nanobelt tangles of nanocrystallized, monoclinic sodium titanate. In a third step, soaking in a simulated body fluid transforms sodium titanate into calcium titanate, by ion exchange in the monoclinic structure. Then, HCA grows and embodies the tangled structure. The interfaces between the different layers seem to be strong enough to prevent from interfacial decohesion. Finally, the role of the titanate structure in the nucleation process of HCA is discussed.
\end{abstract}

KEYWORDS: titanium implants surface, sodium titanate, calcium titanate, hydroxyl carbonated apatite, scanning and transmission electron microscopy,

*corresponding author: egle.conforto@univ-lr.fr. Tel: +33 54645 8617; Fax: +33 546458555. 


\section{Introduction}

Titanium and titanium alloys are widely used for dental and orthopedic implants, due to their high mechanical strength and good bio-compatibility. In addition to these performances it is also crucial to ensure a good osteointegration (Branemark [1]). A current way to achieve a suitable bone-bonding is to deposit a hydroxyapatite (HA) layer with composition similar to the mineral part of the bone, at the implant surface. Plasma spraying is the most used coating technique. However, since it yields to a poor adhesion (Conforto et al [2]), alternative solutions have been developed. Interesting properties have been obtained in this respect after fairly simple chemical surface treatments. The principles of these treatments have been established by Kim et al $[3,4]$. They are based on a chemical activation of the titanium surface during immersion in a $\mathrm{NaOH}$ solution and subsequent heat treatment. On the basis of scanning electron microscopy (SEM) observations and X-rays diffraction (XRD) analyses, these authors proposed the following sequence of transformations. First, the $\mathrm{NaOH}$ treatment generates an amorphous sodium-titanate hydrogel layer, which subsequently transforms into fully crystallized sodium-titanate (indexed as $\mathrm{Na}_{2} \mathrm{Ti}_{5} \mathrm{O}_{11}$ ) and rutile through heat treatments. During in vitro bioactivity tests of the pretreated titanium in simulated body fluid (SBF), $\mathrm{Na}$ ions from the surface were observed to be released, followed by the growth of a hydroxyl carbonated apatite (HCA) layer. Further investigations by Takadama et al [5, 6], including transmission electron microscopy (TEM) observations, yielded more details on the release of $\mathrm{Na}$ ions in SBF prior to HCA nucleation and growth. These authors indeed showed that about $30 \%$ of $\mathrm{Na}$ atoms are replaced by $\mathrm{Ca}$ atoms after 3 days in $\mathrm{SBF}$, which has been interpreted as a partial transformation of the amorphous sodium-titanate into an amorphous calcium-titanate. The possible existence of calcium-titanate has however never been mentioned in further publications. 
Ushida et al [7] subsequently discussed the effect of a more or less pronounced washing in water, on the removal of $\mathrm{Na}$ atoms from the titanate layer. After complete elimination of $\mathrm{Na}$ atoms, and subsequent high-temperature treatment, the sodium titanate layer has been shown to transform into anatase titanium oxide.

The same method has been used and improved by several groups. Wen et al [8], and Jonasova et al [9] aimed to increase the roughness of the initially polished titanium substrate surface by acid etching in $\mathrm{HCl}$, before soaking in $\mathrm{NaOH}$ and SBF. This procedure not only allows obtaining the morphological desired effect but also induces, through the acid etching, the growth of a titanium hydride layer [10]. This may increase the efficiency of the subsequent $\mathrm{NaOH}$ treatment, according to Tanaka et al [11]. The chemical effect involved is however not clearly understood.

Another debated topic is the origin of the easy growth of $\mathrm{HCA}$ induced by $\mathrm{HCl}$ and $\mathrm{NaOH}$ treatments. It has been interpreted in two alternative ways: i) the formation of anatase (and, to a less extent, rutile) nanoparticles, which may enhance the epitaxial growth of HA [7], and ii) the presence of amorphous calcium titanate, which may promote HA growth on account of some chemical affinity $[5,6]$.

Independently, sodium titanate nanobelts, nanorods and nanotubes, have been extensively studied, in view of various applications including ion-exchange capability [12-21]. These structures are grown on $\mathrm{TiO}_{2}$ particles by a process similar to that described above. They nucleate as $\mathrm{Na}_{2} \mathrm{Ti}_{3} \mathrm{O}_{7}$ sodium titanate, after $\mathrm{NaOH}$ treatment, and transform into first $\mathrm{H}_{2} \mathrm{Ti}_{3} \mathrm{O}_{7}$, and then into monoclinic $\mathrm{TiO}_{2}$ or anatase, after various washing procedures (with or without $\mathrm{HCl})$ and heat treatments. These studies have never been compared to those carried out on biomaterials, to the authors' knowledge. They will be described more completely in the discussion paragraph. 
In all cases, the essential problem to be solved is the way by which HCA nucleates and grows on the treated $\mathrm{Ti}$ surface. Up to now, the process steps involved in biomimetic materials obtainment have been basically studied by scanning electron microscopy (SEM) and X-ray diffraction. A single study performed by TEM is reported in the literature by Takadama et al [6], on titanium grids subjected to $\mathrm{NaOH}$ and $\mathrm{SBF}$ treatments. In these experiments, only the outmost part of the titanate structure (after $\mathrm{NaOH}$ treatment) and HCA layer (after soaking in SBF) could be observed and analyzed. Observations below the sample surface, at a depth of several micrometers, are possible only in thin foils prepared in cross-section as in previous studies by Conforto et al [10] and Muller et al [22]. The same method is used in this article to analyze the transformations at the surface of a Ti substrate after etching with $\mathrm{HCl}$ followed by the treatment in a $\mathrm{NaOH}$ solution, and soaking in SBF.

\section{Experimental}

Commercially pure titanium plates with a thickness of $1 \mathrm{~mm}$ were etched using $37 \mathrm{wt} \%$ hydrochloric acid $(\mathrm{HCl})$ under $\mathrm{CO}_{2}$ atmosphere for 2 hours at $50^{\circ} \mathrm{C}$. The goal of this etching is to dissolve the titanium oxide passivation layer, and to generate a homogeneous surface with an average roughness of $4 \mu \mathrm{m}$. The specimens were subsequently soaked in $10 \mathrm{~mol} / \mathrm{l} \mathrm{NaOH}$ aqueous solution at $60^{\circ} \mathrm{C}$ for $24 \mathrm{~h}$, washed with distilled water, and dried at $100^{\circ} \mathrm{C}$. Finally, samples were exposed to SBF solutions with a composition similar to human blood plasma (Table 1) under static conditions at $37^{\circ} \mathrm{C}$ for 2 weeks, in order to obtain a homogeneous apatite layer. SBF solutions were prepared by adding concentrated solutions of $\mathrm{KCl}, \mathrm{NaCl}, \mathrm{NaHCO}_{3}$, $\mathrm{MgSO}_{4} \times 7 \mathrm{H}_{2} \mathrm{O}$, and $\mathrm{KH}_{2} \mathrm{PO}_{4}$, to double distilled water buffered with tris-hydroxymethyl aminimethan and $\mathrm{HCl}$ to $\mathrm{pH}=7.4$ at $37^{\circ} \mathrm{C}$ (Müller et al [23]). Sodium azide $\left(\mathrm{NaN}_{3}\right)$ was added to the solution to inhibit the growth of bacteria. After exposure, the samples were washed in double distilled water. 
Table 1.

\begin{tabular}{lcccccccc}
\hline & $\mathrm{Na}^{+}$ & $\mathrm{K}^{+}$ & $\mathrm{Mg}^{2+}$ & $\mathrm{Ca}^{2+}$ & $\mathrm{Cl}^{-}$ & $\mathrm{HCO}_{3}^{-}$ & $\mathrm{HPO}_{4}^{2-}$ & $\mathrm{SO}_{4}^{2-}$ \\
\hline Blood plasma & 142.0 & $3.6-5.5$ & 1.0 & $2.1-2.6$ & $95.0-107.0$ & 27.0 & $0.65-1.45$ & 1.0 \\
SBF & 142.0 & 5.0 & 1.0 & 2.5 & 126.0 & 10.0 & 1.0 & 1.0 \\
\hline
\end{tabular}

The microstructure and elemental composition were determined by electron microscopy on samples at different steps of the treatment namely i) after $\mathrm{HCl}$ etching, ii) after additional soaking in $\mathrm{NaOH}$ solution, and iii) after exposition of the resulting surface to SBF. Scanning electron microscopy (SEM) observations were carried out using two Philips/FEI microscopes equipped with an EDAX system: XL-30 FEG, operated in the 3-10 kV range, and Quanta 200 ESEM/FEG operated at $20 \mathrm{KV}$ and at variable pressure in the specimen chamber. Transmission electron microscopy (TEM) was performed with two JEOL JEM 2010 and 2011 apparatus operated at $200 \mathrm{kV}$. Bright-field images, and selected area diffraction patterns were obtained from TEM cross-sectional specimens. Diffraction patterns were indexed using conventional methods (see e.g. [24]). To prepare them, two surfaces were joined with epoxy glue. The resulting sandwich was cut perpendicular to the original surfaces, mechanically polished, and thinned to perforation by ion-milling using a GATAN Precision Ion Polishing System - PIPS apparatus, in complete rotation mode. The bombardment was performed using two beams of $\mathrm{Ar}^{+}$ions with energy of $5 \mathrm{keV}$ at low angle $\left(7^{\circ}\right)$, in order to obtain the largest thin area, from the substrate up to the surface.

\section{Results}

\subsection{Samples after etching in $\mathrm{HCl}$}

The SEM image in Fig. 1 shows the rough titanium surface after acid etching. TEM crosssection observations on this kind of sample are similar to those previously published by 
Conforto et al [10] and show the transformation of the Ti substrate surface into a $\mathrm{TiH}_{2-\varepsilon}(\varepsilon<$ $0.1)$ hydride phase, with a face-centered structure $(\mathrm{a}=0.440 \mathrm{~nm})$, over a thickness of several microns. Such a hydride layer can be seen in fig. 3a (cross-sectional TEM image), as part of the structure grown after $\mathrm{HCL}+\mathrm{NaOH}$ treatment. It is constituted of small (few nm in size) heavily deformed grains, containing a high density of dislocations. Its interface with the $\mathrm{Ti}$ substrate is visible at a depth of several microns. The Ti substrate appears as a large-grained material containing a dislocation density lower than $10^{-8} \mathrm{~cm}^{-2}$ (see [10] for more details ${ }^{1}$ ), even close to the interface. No oxide has been detected at the upper surface of the hydride layer, at the scale of TEM observations (resolution of the order of a few nanometers).

\subsection{Samples after $\mathrm{HCl}+\mathrm{NaOH}$ treatments}

Fig. 2a shows the same surface as in fig. 1, after soaking in $\mathrm{NaOH}$ solution. It is covered by tangles of nanofilaments, or nanobelts, separated by distances scaling with the preceding surface roughness size. This structure, already observed by Jonasova et al [9], is clearly different from the more regular one observed by Kim et al [4] and Chosa et al [25], in samples not pre-etched with $\mathrm{HCl}$. The EDS analyses in SEM shown in fig. $2 \mathrm{~b}$ reveal the presence of $\mathrm{Ti}$, $\mathrm{O}$, and $\mathrm{Na}$ atoms. Various measurements indicate that the atomic ratio $\mathrm{Na} / \mathrm{O}$ is constant and equal to $0.14 \pm 0.2$, whereas the atomic ratio $\mathrm{Ti} / \mathrm{O}$ is variable and comprised between 0.5 and 1.4. The latter variation is ascribed to the presence of the titanium-hydride substrate at a variable depth under the surface.

One of these tangles is visible in the cross-section view (figs. 3a and b). This image and many similar ones show that tangles grow preferentially at the tips of the rough Ti-hydride surface. Fig. 3b, where the tip is enlarged, shows that the interface between the hydride layer and the tangled structure is sharp.

\footnotetext{
${ }^{1}$ the contrast in fig. $3 \mathrm{a}$ is generated by surface ripples due to ion milling
} 
The diffraction pattern on the left-hand side of fig. 4 has been taken over the nanobelts selected by the aperture shown in the corresponding image. It is more precise than those previously obtained by Takadama et al [6], where the inner arc of ring could not be resolved ${ }^{1}$. It has been ascribed to $\mathrm{Na}_{2} \mathrm{Ti}_{6} \mathrm{O}_{13}$, in agreement with the measured atomic ratio $\mathrm{Na} / \mathrm{O}=0.13$, and according to JCPDS card $\mathrm{n}^{\circ} 73-1398$. The corresponding structure is monoclinic, with $\mathrm{a}=1.51 \mathrm{~nm}, \mathrm{~b}=$ $0.37 \mathrm{~nm}, \mathrm{c}=0.92 \mathrm{~nm}, \alpha=\gamma=90^{\circ}, \beta=99.5^{\circ 2}$. Slightly different structures and compositions could however be considered as well, as discussed in $\S 4$ below.

The diffraction pattern of the core (right-hand side of fig. 4) is similar to that of the nanobelts, although rings are complete and not well resolved. Since ring diameters are equal to those corresponding to the crystalline nanobelts (in particular the sharpest ones corresponding to (020) planes), it is inferred that the core contains at least a large fraction of nano-crystals, which does not exclude the presence of some amorphous material. The crystallite size is however smaller in the core than in the external part where the nanobelts are clearly visible. It can be remarked that the diffraction pattern in the left-hand side of fig. 4 exhibits arcs of rings typical of a pronounced texture of the corresponding nanograins. Moreover, arcs of ring (200) and (020) are oriented along directions respectively perpendicular and parallel to the average direction of the nanobelts selected by the aperture. Since there is no rotation between image and diffraction pattern, this indicates that the nanobelts grow along the [020] direction. (110) arcs are in the same average direction as (020) ones (namely the corresponding planes are almost parallel) because the unit cell is elongated in the [100] direction (a $>>b)$. Growth along [020] is confirmed in fig. 5 where the nanobelt structure has been imaged in dark field with electrons selected by the aperture shown in the diffraction pattern. In such conditions, crystallites in bright contrast have their (020) planes perpendicular to the arrow joining the transmitted electron beam to the aperture. Since there is no rotation between the diffraction

\footnotetext{
${ }^{1}$ The small-angle diffraction corresponding to this inner ring was not observed on XRD either (Kim et al [4])

${ }^{2}$ The (002) ring cannot be detected owing to its too low intensity
} 
pattern and the image, and since the crystallites are elongated in the same direction as the arrow, it can be inferred that this direction is $[010]^{1}$.

The observation of an essentially nano-crystalline titanate phase contradicts the presence of amorphous titanate and crystalline rutile and/or anatase, postulated in previous investigations (see introduction). The crystalline titanate cannot be introduced during thin foil preparation, because heating above the temperature of the previous treatments $\left(100^{\circ} \mathrm{C}\right)$ is very unlikely during ion milling. Other arguments in favour of a crystalline phase are given in $\S 4-2$ and $\S 4-5$ below.

\subsection{Samples after $\mathrm{HCl}+\mathrm{NaOH}$ treatment and subsequent testing in $\mathrm{SBF}$}

After soaking in SBF, the surface is covered by a new layer, except for very few areas where the tangled structures remain visible (fig. $6 a, b$ ). The droplet shape of this new layer indicates that it nucleates heterogeneously at few sites, and that all nuclei subsequently grow and coalesce until the surface is completely covered. Fig. $6 \mathrm{~d}$ shows the surface of the new layer at a high magnification.

The tangled structure not yet covered by the new layer is seen in cross section TEM, in fig. 7 . Its morphology is quite similar to that observed in fig. 3, before SBF treatment, and it exhibits a similar diffraction pattern. EDS analyses in SEM however show that the concentration of sodium has drastically decreased, and that calcium and phosphorus are detected instead (fig. 6c). The following atomic ratios have been measured: $\mathrm{Na} / \mathrm{O}=0.016 \pm 0.02, \mathrm{Ca} / \mathrm{O}=0.046 \pm$ $0.05, \mathrm{P} / \mathrm{O}=0.019 \pm 0.05$, and $(\mathrm{Na}+\mathrm{Ca}+\mathrm{P}) / \mathrm{O}=0.083 \pm 0.09$. Such a chemical transformation has already been observed by Takadama et al $[5,6,26]$. These authors interpreted it as a partial exchange between $\mathrm{Na}$ and $\mathrm{Ca}$ ions, of order of $30 \%$ after 3 days soaking in SBF. In our case, the exchange is almost completed after 2 weeks.

\footnotetext{
${ }^{1}$ Spherical aberration might introduce a shift of the image of the diffracting grains, but should not change their shape.
} 
Since the largest fraction of the titanate phase is crystalline, these results clearly show that ion exchange takes place in the nanocrystals constituting the tangled structure, not inside an amorphous phase, as postulated by Takadama et al $[5,6,26]$. It is remarkable that this ion exchange takes place with no detectable changes in the monoclinic crystal structure, as the diffraction pattern remains almost the same.

The same tangled structure, covered by the new layer, is seen in fig. 8. This new layer is made of $\mathrm{Ca}$, $\mathrm{P}$, and $\mathrm{O}$ atoms, according to EDS analysis in SEM (fig. 6e). Its structure exactly corresponds to hexagonal hydroxyl carbonated apatite (HCA) according to our previous study (Müller et al [22]). It is interesting to note that HCA does not deeply penetrate through the open space between titanate filaments.

Fig. 9 shows that the transition zone, between titanate and HCA, is smaller than $200 \mathrm{~nm}$. In this transition zone, the rings of the titanate diffraction pattern vanish and those of HA become visible. The possible existence of an amorphous phase in this area is an open question.

Lastly, the adhesion between the different superimposed layers and the substrate can be roughly estimated in the following way. It is well known that the thinning procedure to prepare cross-sectional specimens for TEM considerably weakens the structure, which results in the formation of cracks. Such cracks have actually been observed, through titanate and HCA layers (one of them can be seen in fig. 9). They are however neither at the $\mathrm{Ti}_{-} \mathrm{TiH}_{2-\varepsilon}$ interfaces, nor at the $\mathrm{TiH}_{2-\varepsilon}$-titanate and titanate-HA ones. This indicates that the adhesion between the different layers and the substrate is good. This should however be more accurately measured using more dedicated methods. The case of $\mathrm{Ti}^{-} \mathrm{TiH}_{2-\varepsilon}$ interfaces has already been discussed by Conforto et al [10].

\section{Discussion}

\subsection{Roughness of the titanium hydride surface}


Etching the Ti substrate with $\mathrm{HCl}$ has been introduced in view of obtaining a homogeneously rough surface, on which adhesion is a priori easier (Wen et al [8], Jonasova et al [9]). This procedure has been subsequently shown to induce the transformation of the Ti substrate into a Ti-hydride, over a depth of several micrometers (Conforto et al [10]).

Since our observations show that titanate tangles grow preferentially at the tips of the hydride surface, the roughness is enhanced after the $\mathrm{NaOH}$ treatment. This structure is clearly different from the more uniform one obtained in the absence of $\mathrm{HCl}$ etching, as already shown by Jonasova et al [9]. The role of the hydride layer on the different steps of the process will be discussed in more details in $\S 4-6$.

\subsection{Titanate structure: comparison with previous studies on biomimetic materials}

The structure grown in $\mathrm{NaOH}$ solution (without any subsequent heat-treatment) was investigated by X-ray diffraction by Kim et al [3, 4], and Uchida et al [7]. It has been interpreted as an amorphous sodium titanate hydrogel. Our electron diffraction patterns, showing a well crystallized structure, through a well resolved (200) ring, do not confirm this interpretation. They indicate that the layer contains large amounts of a monoclinic structure, which, taking into account results of local chemical analysis, is likely to be a nanocrystalline sodium titanate of composition $\mathrm{Na}_{2} \mathrm{Ti}_{6} \mathrm{O}_{13}$. Note that the compound $\mathrm{Na}_{2} \mathrm{Ti}_{5} \mathrm{O}_{11}$ proposed by $\mathrm{Kim}$ et al $[3,4]$ is unlikely to exist, according to Bamberger and Begun [27].

We have neither observed rutile, nor anatase, nor amorphous titanate. The absence of any amorphous titanate phase is confirmed by a comparison with the structures described below.

\subsection{Titanate structure - comparison with nanostructures grown on titanium oxyde particles}


Titanate nanostructures have been developed for many different applications (semiconductor devices, photovoltaic cells, photocatalysts, gas sensors etc.). Many structural studies have been published, to which we are going to compare ours.

Nanotubes, nanobelts and nanorods, grown on $\mathrm{TiO}_{2}$ anatase particles subjected to similar $\mathrm{NaOH}$ treatment (slightly higher temperature, comprised between 100 and $180^{\circ} \mathrm{C}$ ) and washing procedure (water and $\mathrm{HCl}$ ) as above, have been observed and studied by Kasuga et al [12], Lan et al [13], Nakahira et al [14], Sun et al [15, 16], Yao et al [17], Wang et al [18], Meng et al [19], Zhang et al [20], Morgado et al [21]. Interesting results have been obtained by TEM, showing nanobelts very similar to those observed in our study. Some of these structures yield diffraction patterns identical to ours (Yao et al [17], Wang et al [18], Chen et al [28], Morgado et al [21]). They have sometimes been ascribed to an anatase structure (Yao et al [17], Wang et al [18]), although important rings like (004) and (105) are missing ${ }^{1}$. Other studies conclude to the existence of $\mathrm{a}_{2} \mathrm{Ti}_{3} \mathrm{O}_{7}$ phase (Chen et al [28], Sun et al [16], Zhang et al [20]). However, as summarized by Morgado et al [21], nanostructures are more likely a monoclinic compound with general formula $\mathrm{A}_{2} \mathrm{Ti}_{\mathrm{n}} \mathrm{O}_{2 \mathrm{n}+1}(\mathrm{~A}=\mathrm{Na}, \mathrm{H}$, and $\mathrm{n}=3-4)$, where the atomic ratio $\mathrm{H} / \mathrm{Na}$ increases with the intensity of the washing process. The formation of such nanostructures is explained by the breaking of $\mathrm{TiO}_{2}$ chemical bonds by $\mathrm{NaOH}$, and the formation of layered entities which possibly transform in nanotubes through a sheet folding mechanism. The resulting compound exhibits high ion-exchange capability, which means that $\mathrm{Na}$ atoms contained in tunnels of the crystalline lattice can easily be removed and replaced by other ones (e.g. H), with no detectable changes in crystalline parameters at the sensitivity level of the electron diffraction technique.

In spite of different substrates and slightly different treatment temperatures, these results are probably transposable to biomimetic materials. The structure deposited by the $\mathrm{NaOH}$ treatment should accordingly consist of fully crystallized $\mathrm{A}_{2} \mathrm{Ti}_{n} \mathrm{O}_{2 n+1}$ nanobelts or nanosheets, growing

\footnotetext{
${ }^{1}$ Only Kasuga et al [12] shows convincing diffraction patterns of anatase
} 
preferentially in (200) planes, and along [020] directions. However, since washing was moderate and performed in water only, and since chemical analyses yield the atomic ration $\mathrm{Na} /$ $\mathrm{O}=0.13$, we estimate that the composition of our nanobelts is close to $\mathrm{Na}_{2} \mathrm{Ti}_{6} \mathrm{O}_{13}$. Since the core of the tangled structure exhibits the same diffraction rings as the nanobelts, it is probably an isotropic tangle of the same nanocrystals.

\subsection{Evolution of sodium titanate upon soaking in $S B F$}

Experimental results show that the tangled structures are almost unchanged upon soaking in SBF, whether they are covered by the HCA layer or not. The diffraction patterns are also similar to those discussed above (see figs. $8 \mathrm{~b}$ and 10, where (200) arcs of ring are visible). Chemical analyses however show that a substantial amount of $\mathrm{Na}$ atoms have been replaced by $\mathrm{Ca}$ atoms, in agreement with Takadama et al $[5,6]$. This substitution can be easily explained by the ion-exchange capability discussed in $\S 4-3$. Note that this mechanism is significantly different from the ion-exchange mechanism between amorphous phases proposed earlier by Takadama et al $[5,6]$. The whole procedure can thus be summarized as follows: crystalline sodium-titanate nanobelts transform partly into hydrogen titanate upon washing, and during the first part of soaking in SBF. Then, calcium atoms are incorporated, which yields crystalline monoclinic titanate nanobelts, of composition $\mathrm{A}_{2} \mathrm{Ti}_{6} \mathrm{O}_{13}(\mathrm{~A}=\mathrm{Ca}, \mathrm{Na}, \mathrm{H})$. The presence of $\mathrm{P}$ atoms can be explained in the same way as in orthorhombic $\mathrm{CaTiO}_{3}$, where calcium atoms can be exchanged with phosphorus ones by a surface mechanism (Coreño and Coreño [29]).

\subsection{Nucleation and growth of HCA in SBF}

It is striking that HA nucleates and grows heterogeneously on the $\mathrm{NaOH}$-treated surface. Since experiments showed that the density of reactive $\mathrm{OH}$ groups that can be considered as potential 
nucleation sites is very high [30], this means that the probability of nucleation per each potential site is very low.

Following Ushida et al [31], many research groups consider that the easiest HCA nucleation should take place on anatase crystallites, owing to a theoretically good epitaxial orientation relationship. Some amounts of anatase have been obtained after heat treatment $\left(600^{\circ} \mathrm{C}\right)$ of the sodium titanate layer, and tested as a substrate for HCA nucleation and growth. Experiments of Ushida et al $[7,31]$ actually showed that growth of HCA on anatase is slightly faster than on sodium titanate (although the effect of a more pronounced washing seems to be more efficient). However, Oh et al [32] showed that the bioactivity of anatase nanotubes is increased by soaking in $\mathrm{NaOH}$, namely by the formation of sodium titanate nano-fibers, which contradicts the above hypothesis. Ushida et al [31] also showed that HA does not grow on amorphous sodium titanate obtained by a sol-gel method, which confirms that sodium titanate must be crystalline in order to be bioactive.

All these results tend to prove that HCA can grow on anatase and nano-crystallized titanate as well. The beneficial role of calcium titanate $\mathrm{CaTiO}_{3}$ in the process of apatite growth has been emphasized by Coreño and Coreño [29] and Ergun et al [33]. Although this titanate has an orthorhombic structure, several of the corresponding conclusions may be transposable. In particular, the partial exchange of $\mathrm{Ca}$ and $\mathrm{P}$ atoms at the surface of the titanate, discussed by Coreño and Coreño [29], seems to play an important role in the apatite growth. Since the probability of HCA nucleation on titanate is low, partly because of a poor orientation relationship, it is necessary to expose a very large free surface to SBF, which is naturally achieved by the nanobelt structure. HCA nucleation on anatase may be easier per unit surface, but the transformation of titanate into anatase may considerably reduce the free surface exposed to SBF, whence an equivalent global efficiency. 
Once nucleated, HCA grows and embodies the remaining titanate structures. Although there is no deep interpenetration of HCA and titanate, the anchoring is sufficiently strong to prevent decohesion, as shown by the aspect of cracks in the thin foil.

\subsection{Role of the intermediate titanium hydride layer}

Our observations show that the $\mathrm{TiH}_{2-\varepsilon}$ layer formed by acid etching is still present after subsequent $\mathrm{NaOH}$ treatment and soaking in SBF. This phase was initially unexpected, the acid etching being performed with the only aim of increasing the surface roughness. Fortunately, its mechanical behavior is surprisingly good, on account of the bad reputation of hydride compounds (Conforto et al [10]). In addition, the titanium/titanium-hydride interface exhibits several epitaxial orientation relationships ensuring good adhesion of the hydride layer onto the Ti substrate (Conforto and Caillard [34]). All these properties contribute to inhibit brittle fracture and subsequent decohesion of the surface layers in the body.

Possible chemical effects of hydrogen atoms contained in the hydride layer, on the subsequent steps of the surface treatment, must also be discussed. These effects should be small, considering that the same $\mathrm{NaOH}$ treatment results in the same sodium titanate nanostructure, for different substrate structures and compositions, namely Ti, Ti-hydride (case of biomimetic materials) and $\mathrm{TiO}_{2}$ powder (case of nanostructure synthesis). However, a different conclusion has been drawn by Tanaka et al [11]. These authors indeed showed that a hydride layer strongly enhances the growth of a porous layer, consisting of Ti oxide and sodium titanate, during soaking in $\mathrm{NaOH}$. In addition, hydrogen atoms coming from the hydride substrate may substitute for $\mathrm{Na}$ atoms of the sodium titanate layer and enhance the substitution of $\mathrm{Na}$ atoms by Ca ones.

\section{Conclusions}


Chemically pretreated titanium surfaces for biomedical applications have been studied by scanning and transmission electron microscopy (SEM and TEM), at different steps of the process. TEM observations on cross-section samples allowed us to investigate the structure of the different layers, as a function of depth, with the following results:

1) $\mathrm{HCl}$ etching generates a rough titanium hydride layer, several micrometers thick, which is still present at the end of the process. This layer has good mechanical and adhesion properties onto the titanium substrate.

2) Subsequent treatment in $\mathrm{NaOH}$ solution induces the growth of a nanocrystalline sodiumtitanate layer. It is monoclinic, with average composition $\mathrm{Na}_{2} \mathrm{Ti}_{6} \mathrm{O}_{13}$, and forms tangles of nanobelts growing in the (100) plane and along the [010] direction. These results are in disagreement with previous ones where sodium titanate was assumed to be amorphous. Sodium-titanate nanobelts are similar to those grown on titanium oxide particles found by other research groups for different applications.

3) Subsequent soaking in simulated body fluid (SBF) transforms sodium-titanate into ( $\mathrm{Na}, \mathrm{Ca}$, P, H)-titanate, with the same monoclinic nanobelt structure and same crystalline parameters. This transformation takes place thanks to the high ion-exchange capabilities of the titanate nanocrystalline structure. HCA then nucleates and grows until the titanate layer is completely covered.

4) HA effective nucleation sites are too sparse to be observed in cross-section TEM. In spite of its low probability of nucleation per unit surface, HA can however grow on Ca-rich titanate because the nanobelt structure offers a very large free surface in contact with the SBF. The transition between titanate and HA takes place over less than $200 \mathrm{~nm}$.

\section{Acknowledgements}


We thank Swiss Federal Institute of Technology-Lausanne for SEM XL-30, Centre Commun d'Analyses of La Rochelle University for SEM Quanta 200 and TEM JEOL 2011 and CEMES-CNRS Toulouse for TEM JEOL 2010 facilities. Two of us (L.M. and F.A.M.) acknowledge Deutsche Forschungsgemeinschaft (DFG) for financial support (MU1803/1). 


\section{References}

[1] Branemark PI. Osseointegration and its experimental background. J. Prosthet. Dent. $1983 ; 50: 399$.

[2] Conforto E, Aronsson BO, Salito A, Crestou C, Caillard D. Rough surfaces of titanium and titanium alloys for implants and prostheses. Mater. Sci. and Eng. 2004;C24: 611.

[3] Kim HM, Miyaji F, Kokubo T, Nakamura T. Preparation of bioactive Ti and its alloys via simple chemical surface treatment. J. Biomed. Mater. Res. 1996;32:409.

[4] Kim HM, Miyaji F, Kokubo T, Nishiguri S, Nakamura T. Graded surface structure of bioactive titanium prepared by chemical treatment. J. Biomed. Mater. Res. 1999;45:100.

[5] Takadama H, Kim HM, Kokubo T, Nakamura T. An X-ray photoelectron spectroscopy study of the process of apatite formation on bioactive titanium metal. J. Biomed. Mater. Res. 2001;55: 185 .

[6] Takadama H, Kim HM, Kokubo T, Nakamura T. TEM-EDS study of mechanism of bonelike apatite formation on bioactive titanium metal in simulated body fluid. J. Biomed. Mater. Res. 2001;57: 441.

[7] Uchida M, Kim HM, Kokubo T, Fujibayashi S, Nakamura T. Effect of water treatment on the apatite-forming ability of $\mathrm{NaOH}$-treated titanium metal. J. Biomed. Mater. Res. (Applied Biomaterials) 2002;63:522.

[8] Wen HB, Liu Q, De Wijn J, De Groot K, Cui FZ. Preparation of bioactive microporous titanium surface by a new two-step chemical treatment. J. Mater. Sci.: Mater. in Medicine 1998;9:121.

[9] Jonasova L, Muller FA, Helebrant A, Strnad J, Greil P. Biomimetic apatite formation on chemically treated titanium. Biomaterials 2004;25:1187. 
[10] Conforto E, Caillard D, Aronsson BO, Descouts P. Crystallographic properties and mechanical behaviour of titanium hydride layers grown on titanium implants. Phil. Mag. 2004;84:631.

[11] Tanaka S, Aonuma M, Hirose N, Tanaki T. The preparation of porous $\mathrm{TiO}_{2}$ by $\mathrm{immersing}$ Ti in $\mathrm{NaOH}$ solution. J. of the Electrochemical Soc. 2003;149:D167.

[12] Kasuga T, Hiramatsu M, Hoson A, Sekino T, Niihara K. Formation of titanium oxyde nanotube. Langmuir 1998;14:3160.

[13] Lan Y, Gao X, Zhu H, Zheng Z, Yan T, Wu F, Ringer SP, Song D. Titanate nanotubes and nanorods prepared from rutile powder. Adv. Funct. Mater. 2005;15:1310.

[14] Nakahira A, Kato W, Tamai M, Isshiki T, Nishio K, Aritani H. Synthesis of nanotube from a layered $\mathrm{H}_{2} \mathrm{Ti}_{4} \mathrm{O}_{9}-\mathrm{H}_{2} \mathrm{O}$ in a hydrothermal treatment using various titania sources. $\mathrm{J}$. of Mater. Sci. 2004;39:4239.

[15] Sun X, Chen X, Li Y. Large-scale synthesis of sodium and potassium titanate nanobelts. Inorganic Chemistry 2002;41:4996.

[16] Sun X, Li Y. Synthesis and characterization of ion-exchangeable titanate nanotubes. Chem. Eur. J. 2003;9:2229.

[17] Yao BD, Chan YF, Zhang XY, Zhang WF, Yang ZY, Wang N. Formation mechanism of $\mathrm{TiO}_{2}$ nanotubes. Appl. Phys. Letters 2003;82:281.

[18] Wang W, Varghese OK, Paulose M, Grimes CA, Wang Q, Dickey EC. A study of the growth and structure of titania nanotubes. J. Mater. Res. 2004;19:417.

[19] Meng X, Wang D, Liu J, Zhang S. Preparation and characterization of sodium titanate nanowires from brookite nanocrystallites. Mater. Res. Bulletin 2004;39:2163.

[20] Zhang S, Peng LM, Chen Q, Du GH, Dawson G, Zhou WZ. Formation mechanism of $\mathrm{H}_{2} \mathrm{Ti}_{3} \mathrm{O}_{7}$ nanotubes. Phys. Rev. Letters 2003;91:256103. 
[21] Morgado E Jr, De Abreu MAS, Pravia ORC, Marinkovic BA, Jardim PM, Rizzo FC, Araujo AS. A study of the structure and thermal stability of titanate nanotubes as a function of sodium content. Solid State Sci. 2006;8:888.

[22] Müller F A, Müller L, Caillard D, Conforto E. Preferred growth orientation of biomimetic apatite crystals. Journal of Crystal Growth 2007;304:464.

[23] Müller L., Müller F.A., Preparation of SBF with different $\mathrm{HCO}_{3}{ }^{-}$content and its influence on the composition of biomimetic apatites 2006, Acta Biomater. 2, 181-189.

[24] Edington J.W. Electron Diffraction in the Electron Microscope. Monographs in Practical Electron Microscopy in Materials Science, vol 2. Philips Technical Library. Mc Millan Press LTD, London, 1975. See also: Andrew K.W., Dyson D.J., Keown S.R. Interpretation of Electron Diffraction Patterns. Hilger and Watts LTD, London, 1967.

[25] Chosa N, Taira M, Saitoh S, Sato N, Araki Y. Characterization of apatite formed on alkaline-heat-treated Ti. J. Dent. Res. 2004;83:465.

[26] Takadama H, Mizuno M, Kim HM, Kokubo T, Nakamura T. TEM-EDS study of process of apatite formation on bioactive Ti-6Al-4V alloy in simulated body fluid. Ceramic Eng. and Sci. Proceedings 2002;23:753.

[27] Bamberger C, Begun G. Sodium titanate: stoichiometry and Raman spectra. J. Am. Ceram. Soc. 1987;70:C48.

[28] Chen Q, Du G., Zhang S, Peng LM. The structure of titanate nanotubes. Acta Cryst. 2002;B58:587.

[29] Coreño J, Coreño O. Evaluation of calcium titanate as apatite growth promoter. J. Biomed. Mater. Res. 2005;75A:478.

[30] Becker I, Hofmann I, Müller FA. Preparation of bioactive sodium titanate ceramics. J. Eur. Ceram. Soc. 2007: 27: 4547. 
[31] Uchida M, Kim HM, Kokubo T, Fujibayashi S, Nakamura T. Structural dependence of apatite formation on titania gels in a simulated body fluid. J. Biomed. Mater. Res. 2002;64A:164.

[32] Oh SH, Finones RR, Daraio C, Chen LH, Jin S. Growth of nanoscale hydroxyapatite using chemically treated titanium oxyde nanotubes. Biomaterials 2005;26:4938.

[33] Ergun C, Liu H, Halloran JW, Webster TJ. Increased osteoblast adhesion on nanograined hydroxyapatite and tricalcium phosphate containing calcium titanate. J. Biomed. Mater. Res. 2006;80A:990.

[34] Conforto E, Caillard D. A fast method for determining favourable orientation relationships and interface planes: application to titanium-titanium hydrides transformations. Acta Mater 2007;55:785.

\section{Table Captions}

Table 1. Comparison of the ionic concentrations in blood plasma and the theoretical one in $\mathrm{SBF}[\mathrm{mmol} / \mathrm{l}]$.

\section{Figure captions}

Fig. 1: SEM view of the sample surface after acid etching in $\mathrm{HCl}$ solution.

Fig. 2: a) SEM view at Accelerating Voltage $(\mathrm{AccV})=3.0 \mathrm{kV}$; and b) EDS spectrum at AccV $=15 \mathrm{kV}$, of the sample surface after acid etching and $\mathrm{NaOH}$ treatment.

Fig. 3: TEM cross-section view of the sample after acid etching and $\mathrm{NaOH}$ treatment. a) Low magnification image showing the interface between the Ti substrate and the hydride layer, and a sodium titanate nanobelt tangle grown on a tip of the hydride surface. b) High magnification view showing the interface between the hydride layer and the nanobelt tangle. 
Fig. 4: Diffraction patterns over the core and the external part of nanobelt tangles. Note the arcs of rings oriented with respect to the corresponding nanobelt direction. Same conditions as in fig. 3.

Fig. 5: Dark-field image of tangled nanobelts with electrons selected by the aperture shown in the diffraction pattern. Note that nanobelts in bright contrast are parallel to the vector connecting the transmitted electron beam and the aperture. Same conditions as in fig. 3 .

Fig. 6: SEM view and EDS analyses of the sample surface after acid etching, $\mathrm{NaOH}$ treatment, and soaking in SBF. a) General view at low magnification showing a small area not completely covered by $\mathrm{HCA}(\mathrm{AccV}=2.0 \mathrm{KV}$, at high vacuum); Next observations were performed under a water vapour pressure of 0.9 mbar: b) Titanate nanobelt tangle not yet covered by HCA, at high magnification $(\mathrm{AccV}=20 \mathrm{KV})$, and c) corresponding EDS spectrum, at the same AccV; d) $\mathrm{HCA}$ at high magnification $(\mathrm{AccV}=7.0 \mathrm{kV})$ and e) corresponding EDS spectrum $(\mathrm{AccV}=$ $15 \mathrm{KV})$.

Fig. 7: TEM cross-section view of the sample after acid etching, $\mathrm{NaOH}$ treatment, and soaking in SBF. a) Titanate nanobelt tangle, in an area not yet covered by HCA, and b) corresponding diffraction pattern.

Fig. 8: TEM cross-section view of the sample after acid etching, $\mathrm{NaOH}$ treatment, and soaking in SBF, in an area covered by HCA. Ca-titanate phase is present between HCA and the titanium hydride layer. Shadows in HCA and Ca-titanate zones are surface ripples formed during ion milling.

Fig. 9: Transition zone between an embedded titanate nanobelt tangle and HCA. Same conditions as in fig. 8 . 


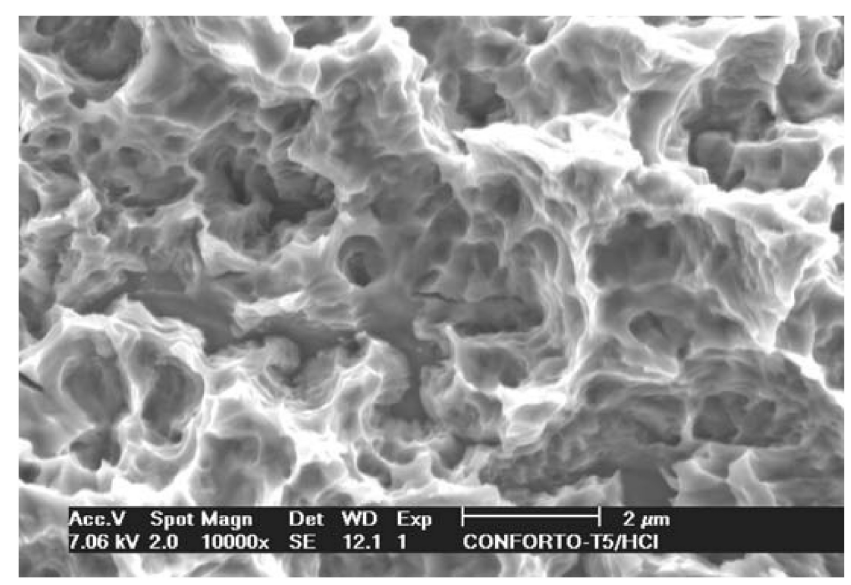

Fig. 1
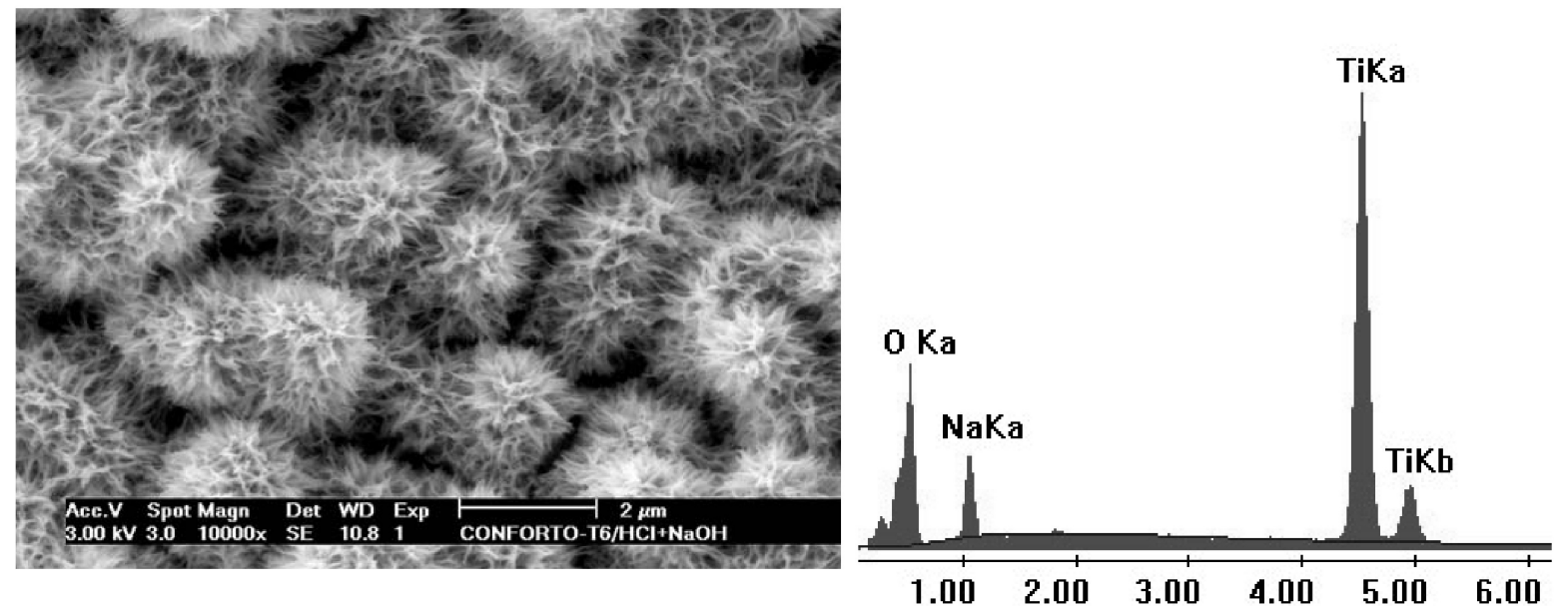

a)

b)

Fig. 2 

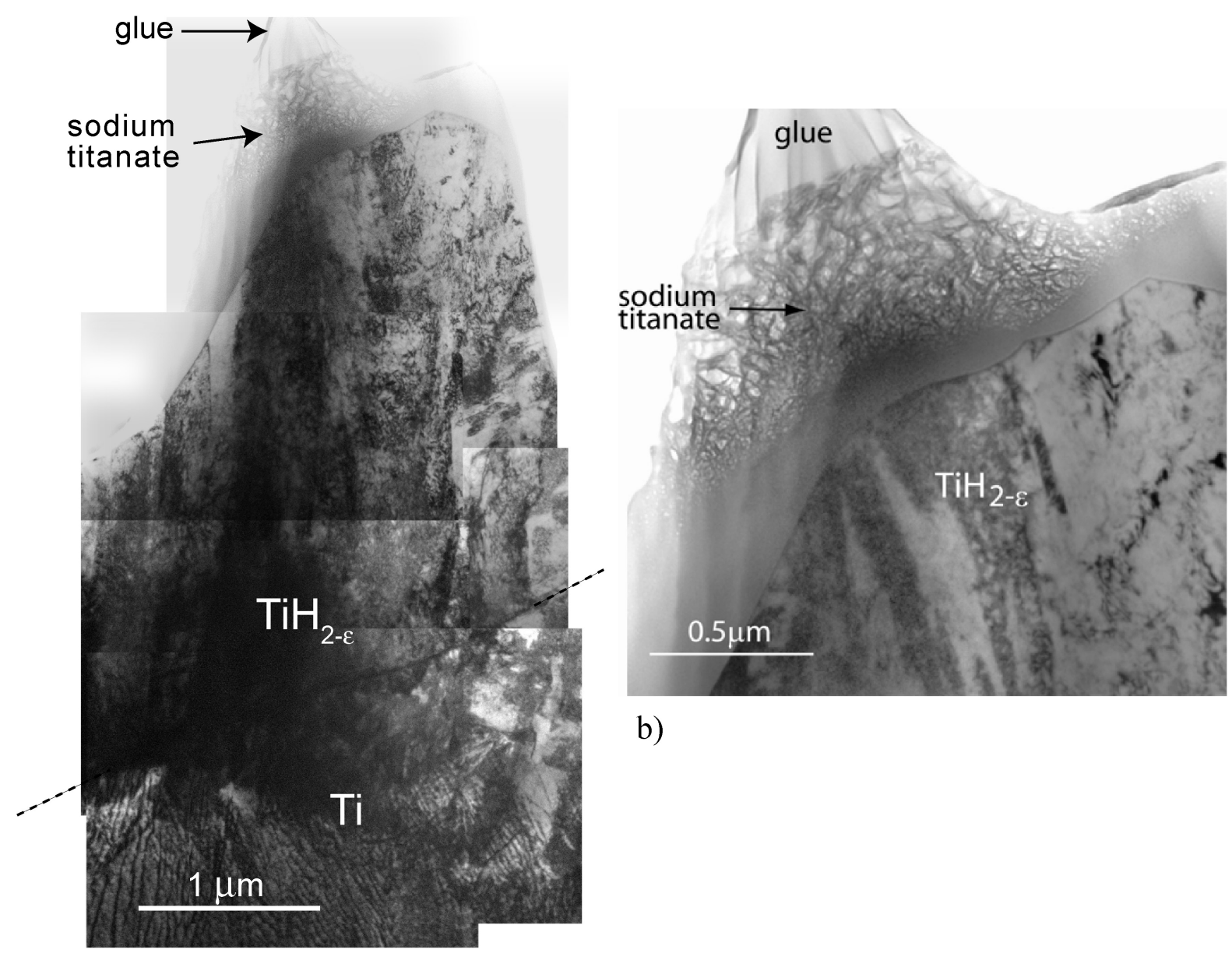

b)

a)

Fig. 3 


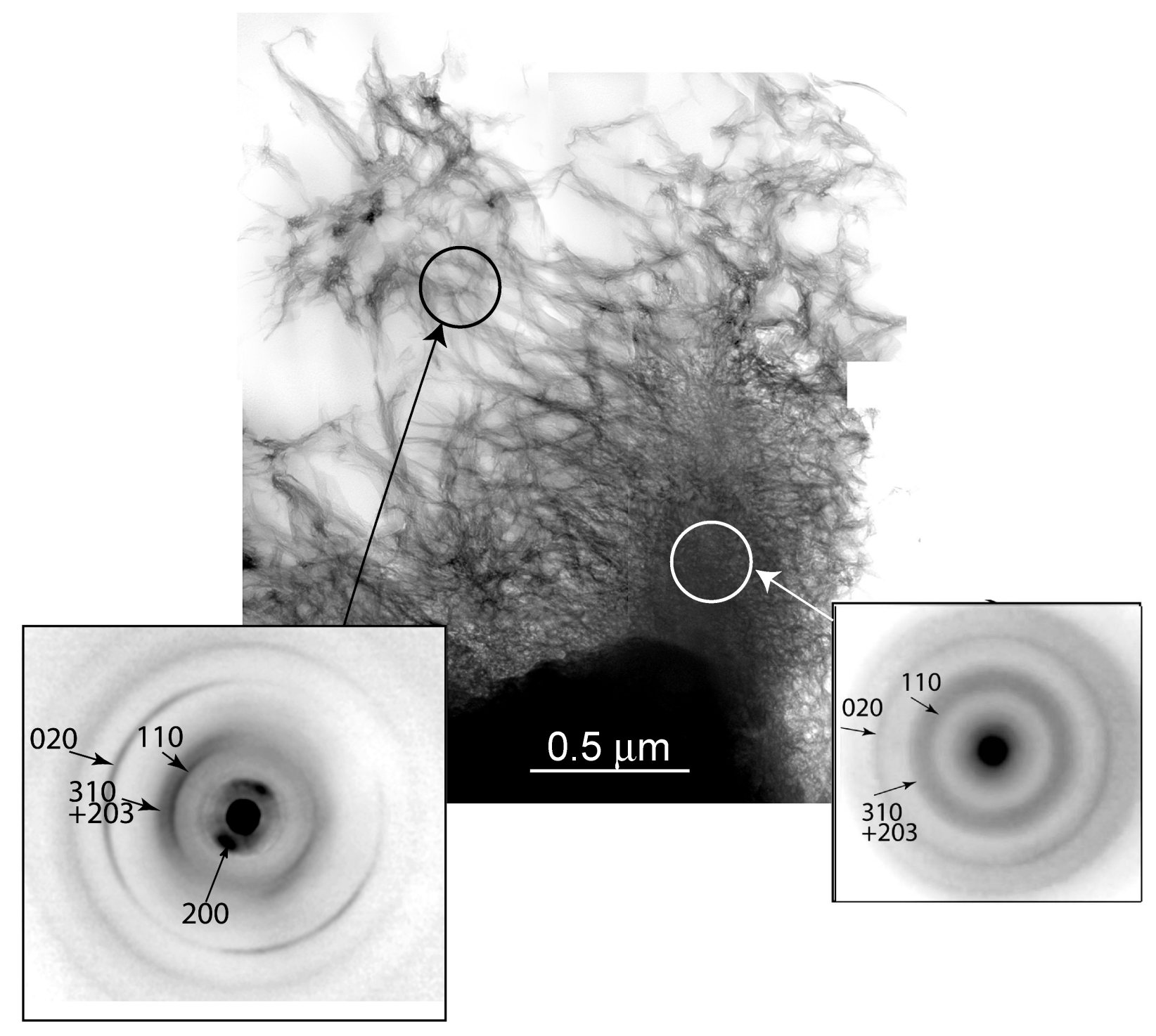

Fig 4 


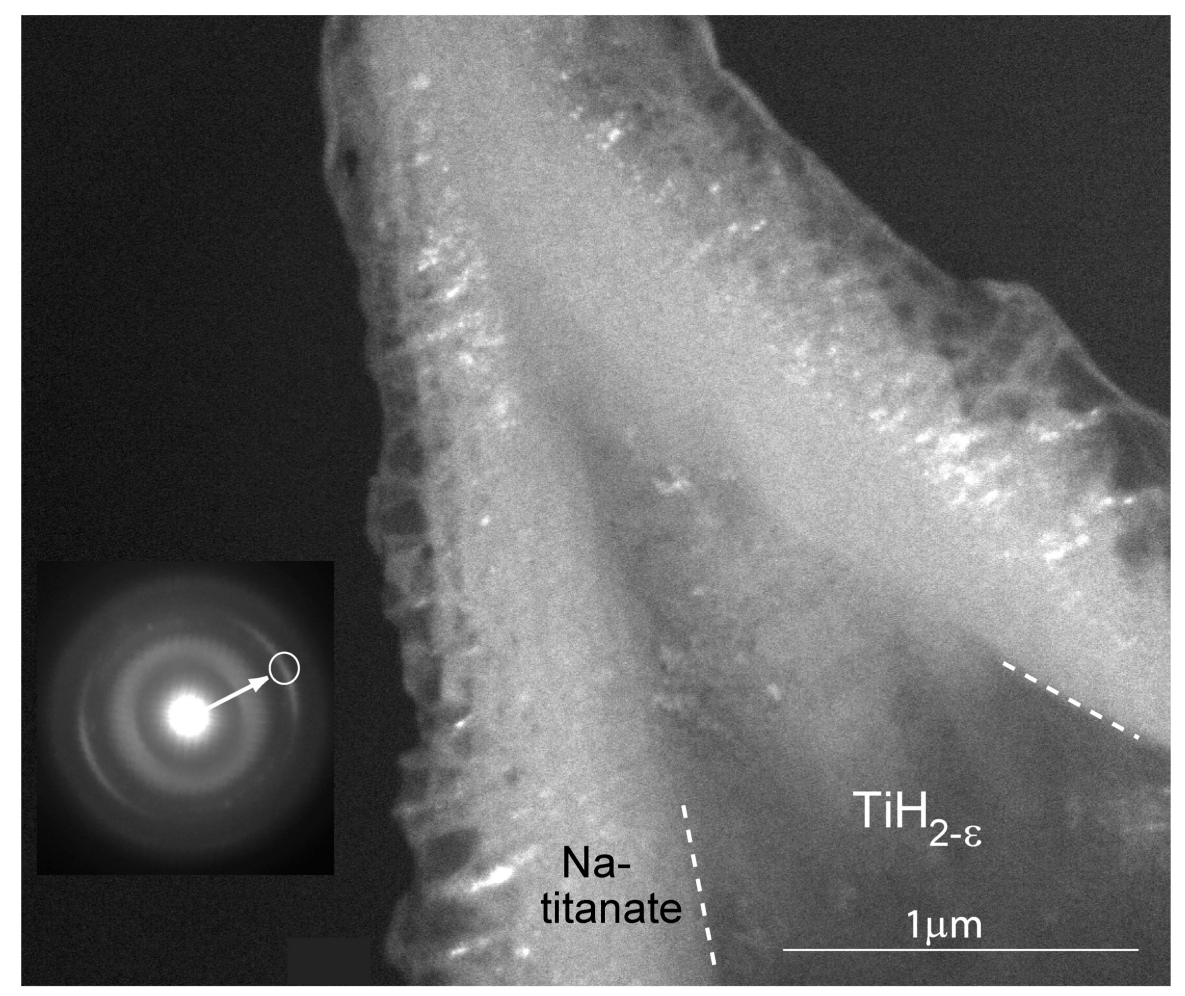

Fig. 5 


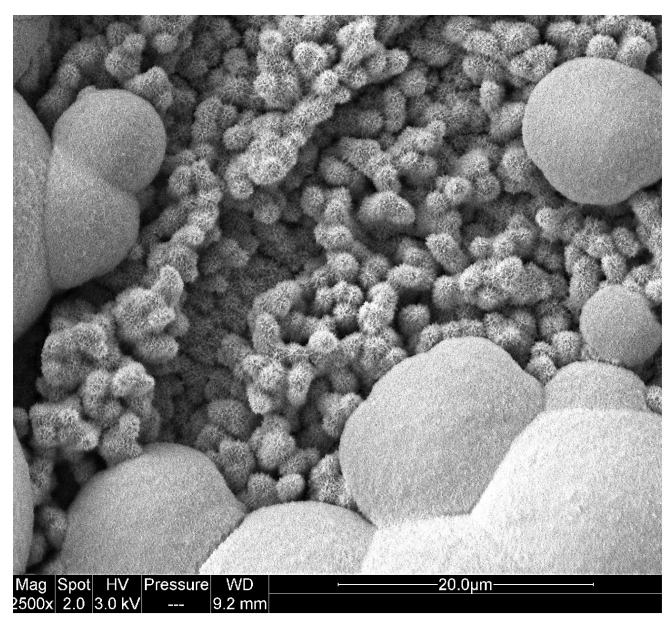

a)

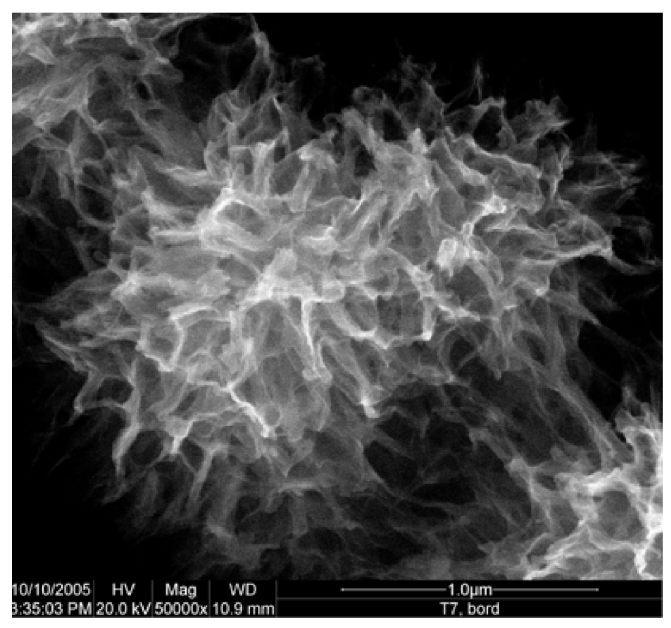

b)
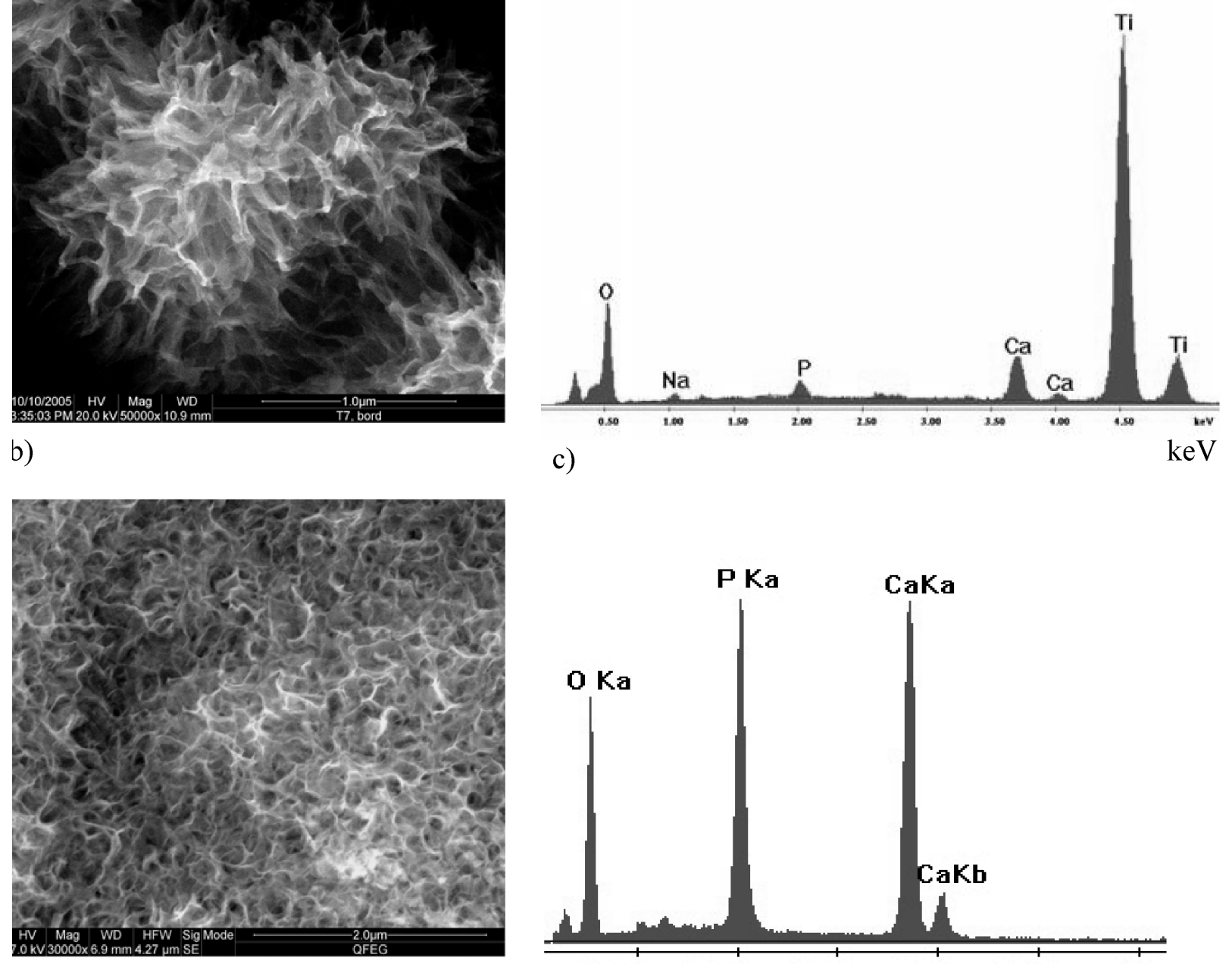

d)

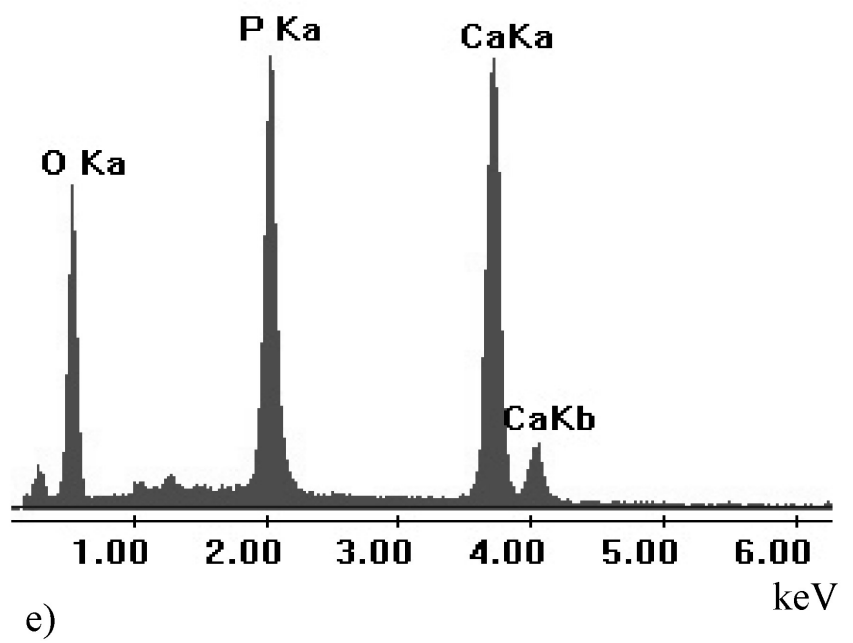

Fig. 6 

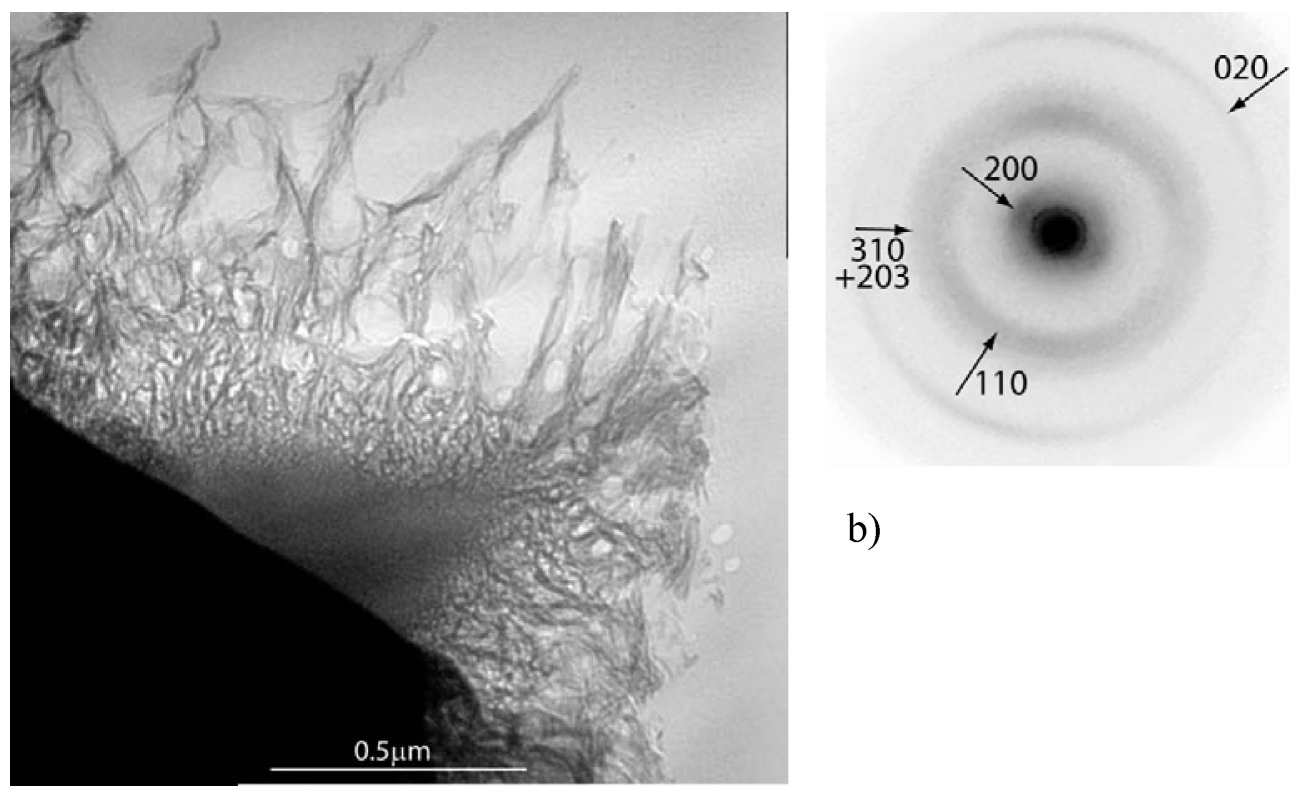

b)

\section{a)}

Fig. 7

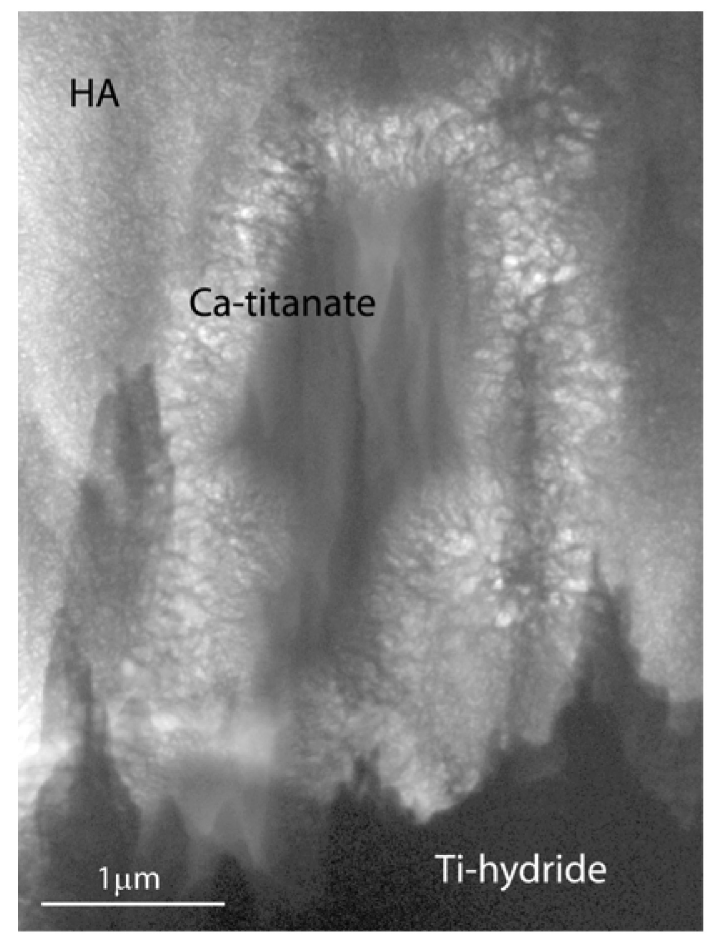

Fig. 8 


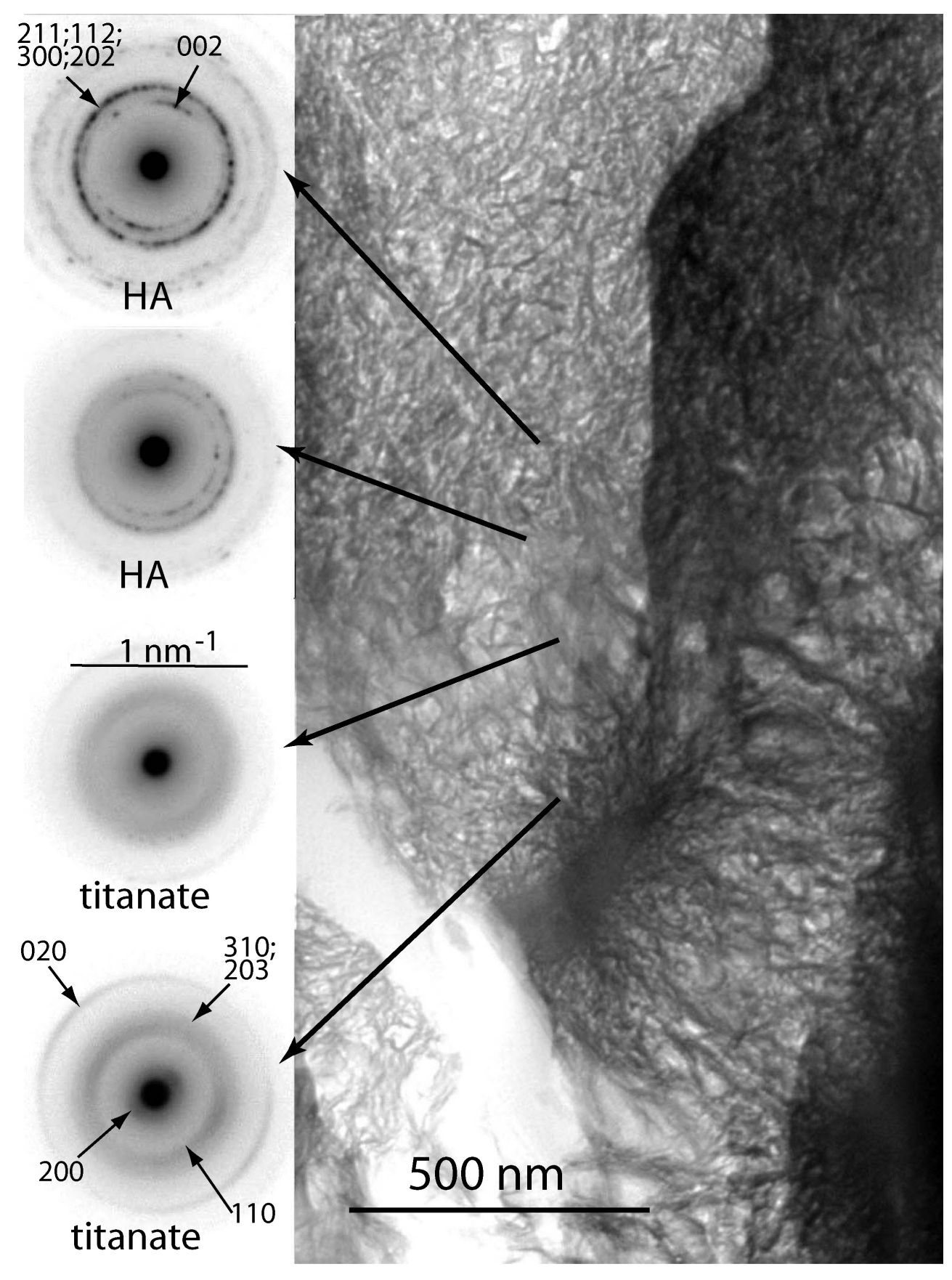

Fig. 9 